\title{
Computational assessment of the effects of a pulsatile pump on toxin removal in blood purification
}

\author{
Ki Moo Lim and Eun Bo Shim*
}

\footnotetext{
* Correspondence:

ebshim@kangwon.ac.kr

1 Department of Mechanical \& Biomedical Engineering, Kangwon National University,

Chuncheon, Kangwon-do 200-

701, Republic of Korea

Full list of author information is

available at the end of the article
}

\begin{abstract}
Background: For blood purification systems using a semipermeable membrane, the convective mass transfer by ultrafiltration plays an important role in toxin removal. The increase in the ultrafiltration rate can improve the toxin removal efficiency of the device, ultimately reducing treatment time and cost. In this study, we assessed the effects of pulsatile flow on the efficiency of the convective toxin removal in blood purification systems using theoretical methods.
\end{abstract}

Methods: We devised a new mathematical lumped model to assess the toxin removal efficiency of blood purification systems in patients, integrating the mass transfer model for a human body with a dialyser. The human body model consists of a three-compartment model of body fluid dynamics and a two-compartment model of body solute kinetics. We simulated three types of blood purification therapy with the model, hemofiltration, hemodiafiltration, and high-flux dialysis, and compared the simulation results in terms of toxin (urea and beta-2 microglobulin) clearance and the treatment dose delivered under conditions of pulsatile and non-pulsatile pumping. In vivo experiments were also performed to verify the model results.

Results: Simulation results revealed that pulsatile flow improved the convective clearance of the dialyser and delivered treatment dose for all three types of therapy. Compared with the non-pulsatile pumping method, the increases in the clearance of urea and beta-2 microglobulin with pulsatile pumping were highest with hemofiltration treatment $(122.7 \%$ and $122.7 \%$, respectively), followed by hemodiafiltration (3.6\% and $8.3 \%$, respectively), and high-flux dialysis (1.9\% and 4.7\%, respectively). EKRc and std Kt/N averaged 28\% and 23\% higher, respectively, in the pulsatile group than in the non-pulsatile group with hemofiltration treatment.

Conclusions: The pulsatile effect was highly advantageous for all of the toxins in the hemofiltration treatment and for $\beta_{2}$-microglobulin in the hemodiafiltration and high-flux dialysis treatments.

\section{Background}

Blood purification systems employing semipermeable membranes are widely used in the treatment of patients with chronic renal failure (CRF). The mechanisms underlying toxin removal in these systems are diffusion and convection [1,2]. As shown in Table 1, the convective mass transfer by ultrafiltration (UF) plays an important role in toxin removal [2].

(c) 2010 Lim and Shim; licensee BioMed Central Ltd. This is an Open Access article distributed under the terms of the Creative Commons BHoWed Central Attribution License (http://creativecommons.org/licenses/by/2.0), which permits unrestricted use, distribution, and reproduction in any medium, provided the original work is properly cited. 
Table 1: Blood purification systems using semipermeable membranes

\begin{tabular}{ll}
\hline Type of blood purification system & Mechanism of toxin removal \\
\hline Conventional hemodialysis & Dialysis \\
HFx & Dialysis + ultrafiltration \\
HF & Ultrafiltration \\
HDF & Dialysis + ultrafiltration \\
\hline
\end{tabular}

Thus, the increase in the UF rate can improve the toxin removal efficiency of the device, eventually requiring less treatment time and cost.

Several experimental studies have indicated that the UF rate in blood purification systems can be increased by using a pulsatile pump instead of a non-pulsatile pump [3,4]. More specifically, our previous study demonstrated that pulsatile flow can generate a greater UF rate than non-pulsatile flow due to the increased transmembrane pressure (TMP) and water permeability of the membrane [3]. However, these studies were limited to basic in vitro experiments to observe the UF efficiency of the dialyser device itself. To our knowledge, in vivo evaluations of the device and its application to patients have not been performed due to difficulties in developing an appropriate animal model. Although animal experiments regarding urea removal during blood purification treatment are relatively easy to perform, serious difficulties exist in establishing an in vivo animal model with a high ? ${ }_{2}$-microglobulin (B2M) concentration. As noted previously by Cameron [5], $\mathrm{B} 2 \mathrm{M}$, which is another significant toxin in blood purification, accumulates slowly in the body under CRF conditions, and therefore, a great deal of time is required for renal failure associated with a high $\mathrm{B} 2 \mathrm{M}$ concentration. However, in vivo analysis is inevitable for a reasonable assessment of the toxin removal efficiency.

Here, we use a theoretical method as an alternative to in vivo experiment to assess the effects of pulsatile pump on toxin removal. For this purpose, we devised a new mathematical model to assess the toxin removal efficiency of blood purification systems in patients, integrating the mass transfer model for a human body [6] with a dialyser in a blood purification system [2]. The present study focused on whether pulsatile flow can improve the delivered treatment dose for patients during blood purification. We performed a simple in vivo clinical test to verify the present simulation model by comparing the simulated urea concentration profile with clinical observations.

Using the mathematical method, we then predicted the dialyser clearances of urea and B2M for various blood purification therapies using pulsatile and non-pulsatile systems. In addition, the treatment dose delivered to patients with CRF was predicted under conditions of pulsatile and non-pulsatile blood purification therapy with a long-term duration of 5 weeks using common blood purification systems, such as hemofiltration (HF), hemodiafiltration (HDF) and high-flux dialysis (HFx).

\section{Methods}

To compare toxin removal efficacy between pulsatile and non-pulsatile systems for the three types of blood purification therapy (i.e., HF, HDF and HFx), we used a computational model with in vitro and in vivo experiments. The in vitro experiment to analyze the contribution of pulsatile flow to the UF rate has already been reported [3] and is summarized in Appendix A1. We identified parameters used in the computational 
model from the results of the in vitro experiment, such as the pressures of the dialyser inlet and outlet of the blood circuit, and the UF coefficient $\left(K_{u f}\right)$ of the dialyser according to the blood pumping rate $\left(Q_{b}\right)$ and pumping type (pulsatile $v s$. non-pulsatile). From these parameters, we theoretically calculated the convective clearance for each type of therapy. By inserting the calculated convective clearance into the computational model, we can simulate the hemodynamic and physiological conditions of hemodialysis patients and assess the effects of pump type and pulsatility on toxin removal efficacy of the patients with a long-term duration. To validate the present computational model, the computation results of urea removal in patients with CRF were compared with the results observed in vivo.

\section{Computational model of the diffusion and convection in the dialyser system}

The mass transfer inside the body that occurs during blood purification therapy can be explained roughly using body solutes and fluid exchange kinetics, as described by Ursino et al. [6]. To simulate the effect of pulsatile flow in a dialyser on the convective clearance of the three types of blood purification therapy, i.e., HF, HDF, and HFx, we modified the dialyser model of Depner and Garred [2] for each therapy and combined this with the human body model of Ursino et al. The human body model is explained briefly below, and the dialyser model for each therapy is explained in the following two sections.

Body fluid consists of three compartments (intracellular, interstitial and plasma) in the model, and two compartments (intracellular and extracellular) are used to formulate the kinetics of the solutes. Fluid exchange between the intracellular and interstitial compartments results from their osmotic pressure difference, whereas oncotic and hydrostatic pressure gradients induce fluid transfer between the interstitial fluid and plasma. Fluid exchange between the plasma and dialysate is determined by the UF rate, which is the product of transmembrane pressure through the dialyser membrane and UF coefficient of the dialyser. Diffusion is the main mechanism of solute movement into and out of cells. In the extracellular compartment, the solute kinetics is influenced by mass transfer through the dialyser in a blood purification system. Here, the model variables related to solutes include urea and B2M as markers of small and large molecular toxins, respectively, and other important electrolytes (sodium, potassium and chloride) and proteins. As the meaningful variables were urea and B2M, we discuss only the variations in urea and B2M, which are markers of uraemic toxins. A schematic diagram of the present mathematical model is shown in Figure 1. A detailed explanation of the governing equations and parameters adopted from Ursino et al. [6] is presented in Appendix A2 and Table 2, respectively.

\section{Convective toxin clearance for HF, HDF and HFx treatments}

HF therapy works by passing the patient's blood through a dialyser that filters out waste products and water and then adds replacement fluid before returning the blood to the body. The replacement fluid maintains fluid volume in the blood and provides electrolytes. The only mechanism of toxin removal in HF therapy is convective transport through the dialyser membrane. HDF is a method that combines hemofiltration and counter-current dialysis for diffusive transport. The dialysate flows in the direction opposite the blood flow in order to maintain the maximum concentration gradient across the membrane, and the counter-current flow condition increases the efficiency of dialysis. Consequently, HDF uses both replacement fluid and dialysate solution, and the mechanism of toxin removal in HDF is both convective and diffusive transport. HFx 
Table 2: Parameter assignment of the mathematical model

\begin{tabular}{lllll}
\hline & Definition & Value & Units & Ref. \\
\hline$K O A_{\text {urea }}$ & $\begin{array}{l}\text { Diffusive mass transfer } \\
\text { coefficient of the dialyser (urea) }\end{array}$ & 967 & $\mathrm{~mL} / \mathrm{min}$ & Specs. \\
$K O A_{B 2 M}$ & $\begin{array}{l}\text { Diffusive mass transfer } \\
\text { coefficient of the dialyser (B2M) }\end{array}$ & 290 & $\mathrm{~mL} / \mathrm{min}$ & Specs. \\
$S_{\text {urea }}$ & $\begin{array}{l}\text { Dialyser sieving coefficient } \\
\text { (urea) }\end{array}$ & 1 & & Specs. \\
$S_{B 2 M}$ & $\begin{array}{l}\text { Dialyser sieving coefficient } \\
\text { (B2M) }\end{array}$ & 0.8 & & Specs. \\
$\eta_{\text {urea }}$ & Transfer coefficient (urea) & 0.77 & $\mathrm{~L} / \mathrm{min}$ & 6 \\
$\eta_{B 2 M}$ & Transfer coefficient (B2M) & 0.077 & $\mathrm{~L} / \mathrm{min}$ & 2 \\
$k_{f}$ & Water-transfer coefficient & 0.24 & $\mathrm{~L} 2 / \mathrm{min} / \mathrm{mmol}$ & 6 \\
$\beta_{\text {urea }}$ & Equilibrium ratio (urea) & 1 & & 6 \\
$\beta_{B 2 M}$ & Equilibrium ratio (B2M) & 1 & & Est. \\
$R$ & Plasma water fraction & 0.94 & & 6 \\
$G$ & Urea generation rate & 6.24 & $\mathrm{mg} / \mathrm{min}$ & 2
\end{tabular}

More detailed cellular and vascular parameters were described previously by Ursino et al. [6]. Est., estimated; Specs., from the FX60 dialyser specifications.

therapy also provides both convective and diffusive transport simultaneously, but it uses only dialysate solution, not replacement fluid. The pressure distribution along the length of the dialyser in both the blood and dialysate compartment is an important factor in convective transport. At the dialyser inlet of the blood circuit is a region of filtration, as the blood pressure exceeds the dialysate pressure. Backfiltration occurs at the region of the dialyser outlet of the blood circuit, as the dialysate pressure exceeds the blood pressure.

Convective toxin clearance is a direct input parameter for the present simulation code and is computed separately for each blood purification therapy by computing the UF rate. The UF rate can be calculated as the product of the mean transmembrane pressure (TMPm) and UF coefficient, shown in the following equations:

$$
\begin{aligned}
& \text { TMPm }=\frac{P_{b, \text { in }}+P_{b, o u t}}{2}-\frac{P_{d, \text { in }}+P_{d, \text { out }}}{2} \\
& Q_{f}=K_{u f} \cdot T M P m
\end{aligned}
$$

where $P_{b, \text { in }}$ and $P_{b, \text { out }}$ are the mean pressures at the dialyser inlet and outlet of the blood circuit, respectively, $P_{d, i n}$ and $P_{d, o u t}$ are those of the dialysate circuit, $K_{u f}$ is the UF coefficient of the dialyser and $Q_{f}$ is the UF rate.

For the HF treatment, $P_{d, \text { in }}$ and $P_{d, \text { out }}$ were set to zero and $P_{b, \text { in }}$ and $P_{b, \text { out }}$ were obtained from the in vitro reference data. Based on these pressures, TMPm was computed using Eq. (1). We calculated the UF rate $\left(Q_{f}\right)$ in Eq. (2) from the UF coefficient obtained from the in vitro experiment and the TMPm. Then, the convective toxin clearance for the HF treatment was calculated with Eq. (A11) shown in the Appendix using the UF rate. 


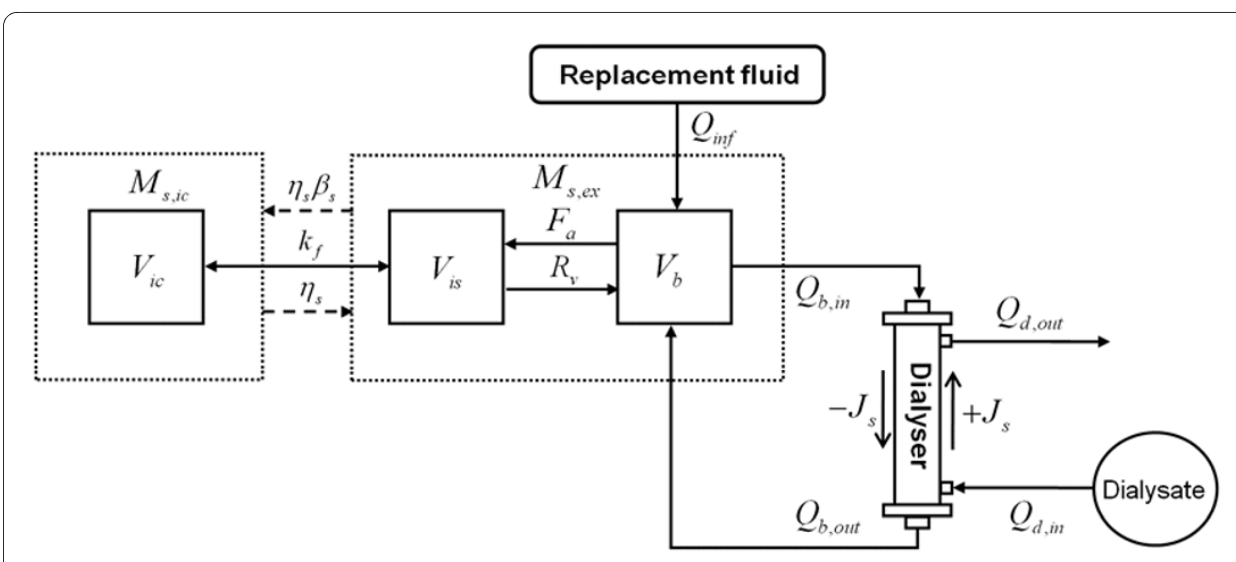

Figure 1 Schematic diagram of the mathematical model for a patient with the blood purification system. There are two compartments for solute kinetics and three compartments for fluid dynamics inside body. (-----), solute transfer; (---), fluid transfer; ic, intracellular; is, interstitial; $p l$, plasma;ex, extracellular; $b$, blood; $d$, dialysate; $\eta_{s^{\prime}}$ mass transfer coefficient of solute sbetween the intracellular and extracellular compartments; $\beta_{s^{\prime}}$ equilibrium ratio of solute $s ; V$, compartment volume; $M_{s^{\prime}}$ mass of the solute $s ; Q_{\text {infi }}$ flow rate of replacement fluid; $J_{s^{\prime}}$ removal rate of solute $s$ across the dialyser; $R_{v^{\prime}}$ fluid reabsorption rate at the venous capillaries; $F_{a^{\prime}}$ fluid filtration rate at the arterial capillaries; $k_{f}$ osmotic filtration coefficient at the cellular membrane; $Q$, extracorporeal flow rate; in, inlet; out, outlet. Modified from Ursino et al. [6] and Depner and Garred [2].

In the HDF treatment, both diffusive and convective clearances exist simultaneously. We set the dialysate flow rate to $300 \mathrm{~mL} / \mathrm{min}$ as in the in vitro experiments. Diffusive toxin clearance for the HDF treatment can be computed from Eq. (A8) in the Appendix using the specified values of the blood and dialysate flow rate through the dialyser. Based on the pressures and UF coefficients obtained from the in vitro experiments, UF rate was calculated using Eq. (1) and (2). Then, convective toxin clearance for the HDF treatment was calculated using Eqs. (A9) and (A10) shown in the Appendix.

HFx treatment also uses convection and diffusion simultaneously for toxin removal but does not use replacement fluid, in contrast to other blood purification systems. Therefore, to maintain a constant body fluid volume as in the other therapies, the net UF should be zero by controlling the UF and backfiltration. In this HFx model, we set the mean pressure at the dialyser inlet and outlet of the dialysate circuit equal to those in the blood circuit to assign a zero net UF. The procedure to compute the UF rate is presented in Eqs. (A12)-(A15) in the Appendix. Convective toxin clearance for the HFx treatment is also calculated in Eq. (A11) in the Appendix using the UF rate.

In vivo test for model validation

In vivo experiments were performed to validate the present computational model. First, we measured urea removal during blood purification in the dialysis unit of Kang's Clinic in Seoul, Republic of Korea, during ten hemodialysis sessions using the AK95 roller pump. Detailed conditions of the clinical measurements are presented in Table 3. Then, we performed model simulations under conditions almost identical to the clinical settings with respect to patient body weight, diffusive mass transfer coefficient of dialyser for urea $\left(\mathrm{KoA}_{u}\right)$, UF rate and blood and dialysate flow rates. The computed results of urea removal were then compared with the in vivo clinical data.

\section{Simulation models}

The calculated convective toxin clearance was applied to the proposed mathematical model. Three blood purification therapies (i.e., HF, HDF and HFx) were simulated for both pulsatile and non-pulsatile systems according to the blood flow rate. Then, we com- 
pared the total performances of urea and B2M for the pulsatile system with those for the non-pulsatile system.

Next, we performed long-term simulations to compare the delivered treatment dose of the pulsatile system with that of the non-pulsatile system. A treatment schedule of three times per week, $4 \mathrm{~h}$ per treatment, was applied to each system. The EKRc and the standard (std) $K t / V$ indices were used to quantify the treatment dose. Here, the EKRc and std $K t / V$ reflect the time-averaged concentration (TAC) and mean pre-treatment concentration (MPC), respectively [7-9]. According to the results of previous studies [9], the required minimum values of EKRc and std $K t / V$ for an anuric patient with a total body water volume of $40 \mathrm{~L}$ are $11 \mathrm{~mL} / \mathrm{min}$ and 2, respectively. The indices are calculated using the time-varying urea concentration profiles in the plasma compartment during 5 weeks of simulation. The indices are defined in the Appendix.

\section{Results}

Parameter estimation and in vivo clinical test

The previous in vitro experimental result was used to determine the parameters of the computational model, such as the inlet and outlet pressures of the blood circuits, and the UF coefficient of the dialyser according to the blood pumping rate and pump type. Figure 2(a) shows the pressures at the dialyser inlet and outlet of the blood circuit according to the blood flow rate and pump type. Under the same mean flow rate, the dialyser inlet pressure of the pulsatile pump was higher than that of the non-pulsatile pump, whilst the dialyser outlet pressure was almost identical for both pulsatile and non-pulsatile pumps. Figure 2(b) shows the derived UF coefficient according to the blood flow rate and pump type. The UF coefficient of the pulsatile pump was higher than that of the non-pulsatile pump, and the difference in the UF coefficient between the two pumps decreased with increasing blood flow rate.

As described in the Introduction, urea removal during blood purification was monitored in vivo for short treatment times. We compared the measured data with computational results. As shown in Figure 3, the simulated plasma urea concentration profile was very similar to the clinical results; both showed a concentration rebound phenomenon after $4 \mathrm{~h}$ of treatment.

Toxin removal dynamics

Here, HF, HDF and HFx were simulated according to the pump type and blood flow rate. Then, we assessed the effects of the pump type on the toxin clearance performances for urea and $\mathrm{B} 2 \mathrm{M}$.

Table 3: Patient conditions and system settings for in vivodialysis experiments

\begin{tabular}{lll}
\hline Parameters & Value & Dimension \\
\hline Number of patients & 18 & \\
Patient body weight & $73 \pm 9$ & $\mathrm{~kg}$ \\
Predialysis urea concentration & $105 \pm 12$ & $\mathrm{mg} / \mathrm{dL}$ \\
$\mathrm{KoA}_{\mathrm{u}}{ }^{*}$ & 967 & $\mathrm{~mL} / \mathrm{min}$ \\
Blood flow rate & 300 & $\mathrm{~mL} / \mathrm{min}$ \\
Dialysate flow rate & 500 & $\mathrm{~mL} / \mathrm{min}$ \\
UF rate & 0.5 & $\mathrm{~L} / \mathrm{h}$ \\
Treatment time & 4 & $\mathrm{~h}$ \\
\hline
\end{tabular}



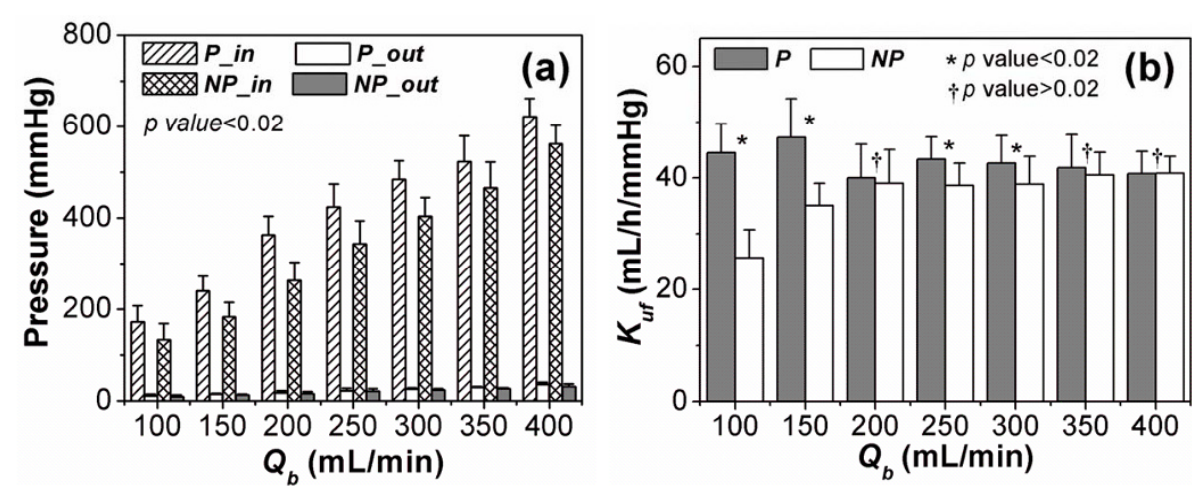

Figure 2 Variations in dialyser pressure and UF coefficients. Variation in (a) the pressure at the dialyser inlet and outlet ( $p<0.02$ for differences between $P_{-}$in and NP_in) and (b) the UF coefficient of the dialyser $(p<0.02$ for differences between $P$ and $N P$ ) for $Q_{b}=100,150,250$, and 300 according to pump type and the mean blood flow rate. $P_{-}$in and $P_{-}$out are the pressures at the dialyser inlet and outlet, respectively, in the pulsatile pump group. NP_in and NP_out are the pressures at the dialyser inlet and outlet in the non-pulsatile pump group. $K_{u f}$ indicates the UF coefficient of the dialyser, $P$ and NP are the UF coefficients in the pulsatile and non-pulsatile groups, respectively, and $Q_{b}$ indicates the mean blood flow rate.

Figure 4(a) shows the percentage increase in computed urea clearance of the pulsatile pump relative to that of the non-pulsatile pump according to the blood flow rate and therapy. The increase in toxin clearance was highest in the HF treatment followed by the HDF treatment over the entire range of flow rates computed (100-400 $\mathrm{mL} / \mathrm{min})$. In the HF treatment, pulsatile pumping increased the urea clearance by a maximum of $122.7 \%$ at a blood flow rate of $100 \mathrm{~mL} / \mathrm{min}$ and a minimum of $10.5 \%$ at a blood flow rate of 400 $\mathrm{mL} / \mathrm{min}$. The percentage increase in urea clearance was reduced with increases in the blood flow rate in HF. In HDF, urea clearance showed a maximum increase of $3.6 \%$ at a blood flow rate of $300 \mathrm{~mL} / \mathrm{min}$ and a minimal increase of $0.05 \%$ at a blood flow rate of $100 \mathrm{~mL} / \mathrm{min}$. In HFx, urea clearance showed maximum and minimum increases of $1.9 \%$ and $0.03 \%$ at blood flow rates of $300 \mathrm{~mL} / \mathrm{min}$ and $100 \mathrm{~mL} / \mathrm{min}$, respectively.

Figure 4(b) shows the percent increase in B2M clearance by pulsatile pumping for the three types of therapy. The HF treatment showed the largest clearance increment followed by the HDF treatment over the entire range of flow rates examined, similar to the case of urea clearance. In HF, the percent increase of B2M clearance was identical to that of urea clearance throughout the entire flow rate. In HDF, the B2M clearance increased by a maximum of $8.3 \%$ at a flow rate of $300 \mathrm{~mL} / \mathrm{min}$ and a minimum of $2.2 \%$ at a flow rate of $100 \mathrm{~mL} / \mathrm{min}$. In $\mathrm{HFx}$, the B2M clearance showed maximum and minimum increases of $4.7 \%$ and $1.5 \%$ at flow rates of $300 \mathrm{~mL} / \mathrm{min}$ and $100 \mathrm{~mL} / \mathrm{min}$, respectively. In the cases of the HDF and HFx treatments, the increase in B2M clearance by pulsatile pumping was larger than that of urea clearance.

\section{Comparison of treatment doses}

Figure 5 shows the computed EKRc and std $K t / V$ indices for 12-h weekly treatments in the three types of therapy over the entire range of flow rates examined $(100-400 \mathrm{~mL} /$ $\min )$. For the HF treatment, EKRc and std $K t / V$ were on average $28 \%$ and $23 \%$ higher in the pulsatile group than in the non-pulsatile group, respectively. However, the superiority of the pulsatile pump was not remarkable in the HDF or HFx treatments, with increments of $<2 \%$ in both treatments. 


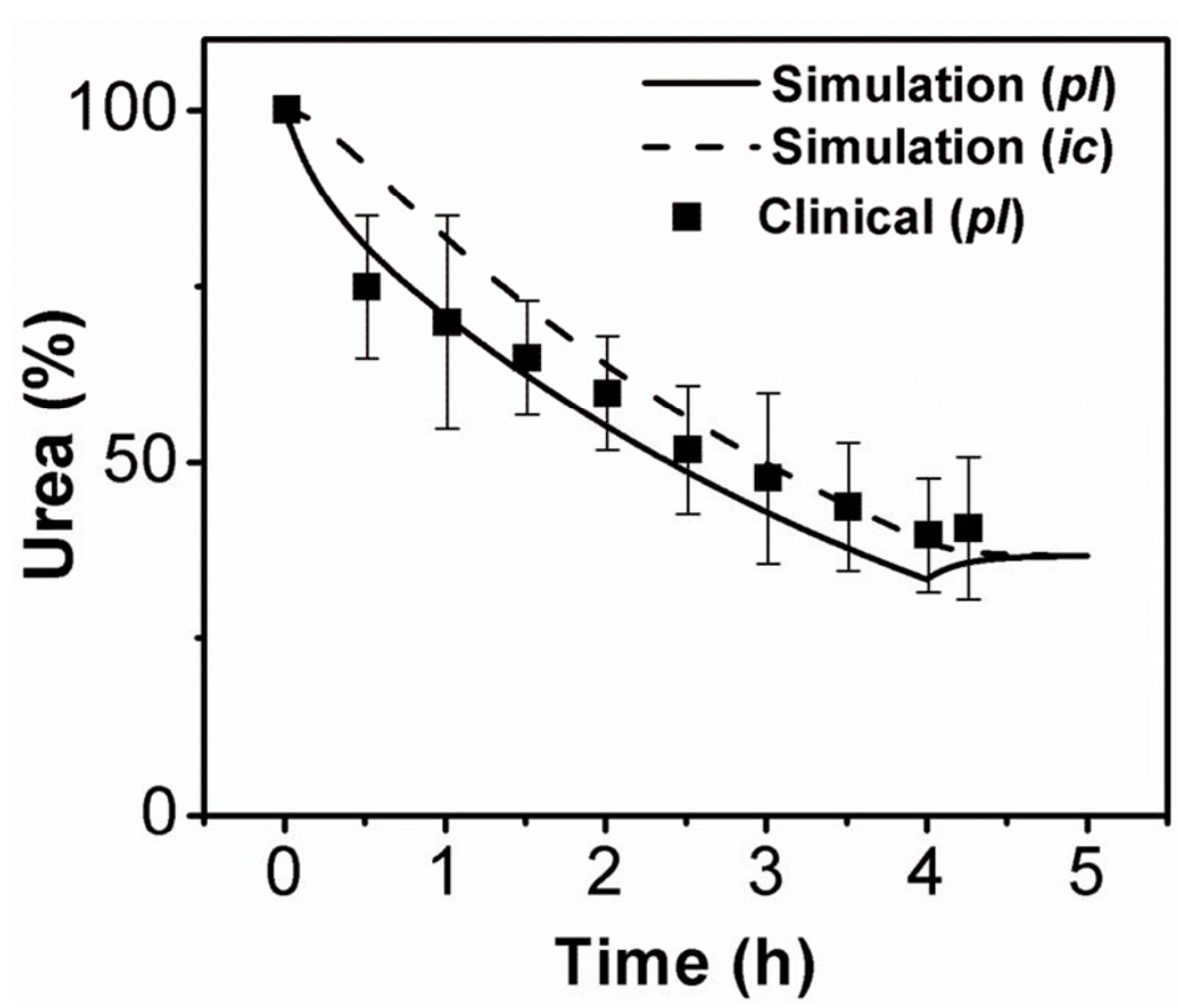

Figure 3 Comparison of the simulated results with the in vivo experiment. Percent changes of the intracellular urea concentration (------) and plasma urea concentration (----) in the simulation and in vivo experiment (ם).

To satisfy the adequacy line of EKRc $\geq 11.0$ and std $K t / V \geq 2.0$, the range of the adequate blood flow rate was $>200 \mathrm{~mL} / \mathrm{min}$ for the HDF and HFx treatments under both pulsatile and non-pulsatile conditions. However, for the HF treatment, the ranges of blood flow rate to satisfy the adequacy line of EKRc $\geq 11.0$ were $>300 \mathrm{~mL} / \mathrm{min}$ and 400 $\mathrm{mL} / \mathrm{min}$ under pulsatile and non-pulsatile conditions, respectively. In addition, the ranges of the blood flow rate for the HF treatment to guarantee the adequacy line of std
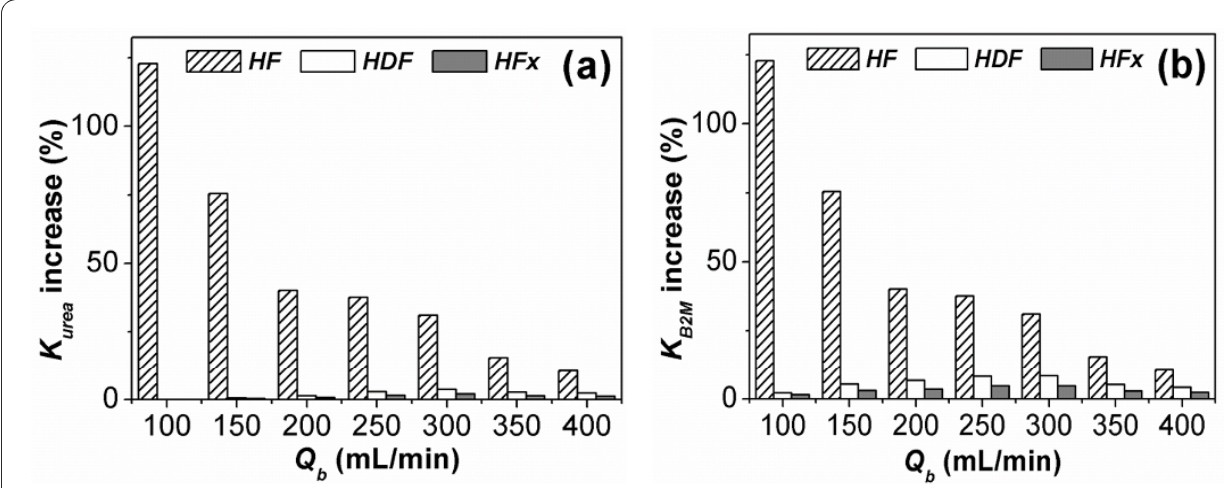

Figure 4 Percent increase of dialyser clearance by pulsatile pumping. Difference in dialyser clearance between the pulsatile pump group and non-pulsatile pump group according to treatment type and mean blood flow rate. (a) Percent increase in urea clearance. (b) Percent increase in B2M clearance. $K_{\text {urea }}$ and $K_{B 2 M}$ are the dialyser clearances for urea and $B 2 M$, respectively, and $Q_{b}$ indicates the mean blood flow rate. 
$K t / V \geq 2.0$ were $350 \mathrm{~mL} / \mathrm{min}$ and $400 \mathrm{~mL} / \mathrm{min}$ under pulsatile and non-pulsatile conditions, respectively.

\section{Discussion}

In our previous in vitro study, we demonstrated the superiority of a pulsatile pump relative to a non-pulsatile or weak pulsatile pump in terms of the TMP and UF coefficients of the dialyser [3]. Compared to the non-pulsatile pump, the pulsatile pump induced a higher dialyser pressure, such as dialyser inlet pressure and TMP, and a higher UF coefficient at the same mean blood flow rate. The dialyser inlet pressure is influenced by the flow impedance. Therefore, it is clear that the flow wave-form through the dialyser produced by the pulsatile pump induced higher flow impedance than did that produced by the roller pump. As both blood pumps are flow generators and not pressure generators, the pump inducing the higher impedance develops a higher fluid power to maintain the target flow rate, and consequently produces a higher dialyser inlet pressure, which in turn results in a higher TMPm and UF rate. In addition, an instantaneous high pressure developed as the pulsatile pump increased the UF coefficient due to the reduction of membrane layering. However, the difference in the UF coefficient between pulsatile and roller pumps decreased as the pumping rate increased. This implies that an effective pumping frequency exists for reducing membrane layering. In other words, a greater pumping frequency makes the system less efficient in terms of the UF coefficient. These results were limited to basic in vitro experiments to determine the UF efficiency of the dialyser device itself.

In this study, theoretical evaluations of the apparatus and its application in patients were performed to assess the effects of pulsatile flow on the efficiency of convective toxin removal in blood purification systems. For this purpose, we devised a new mathematical model, integrating the mass transfer model for the human body [6] with that for the dialyser (Figure 1). For verification of the model, we simulated conventional hemodialysis therapy for $4 \mathrm{~h}$, and the computed urea concentration profile inside the body was compared with clinical observations (Figure 3). Using the verified model, we theoretically predicted the effect of pulsatile pumping on convective toxin clearance (Figure 4)
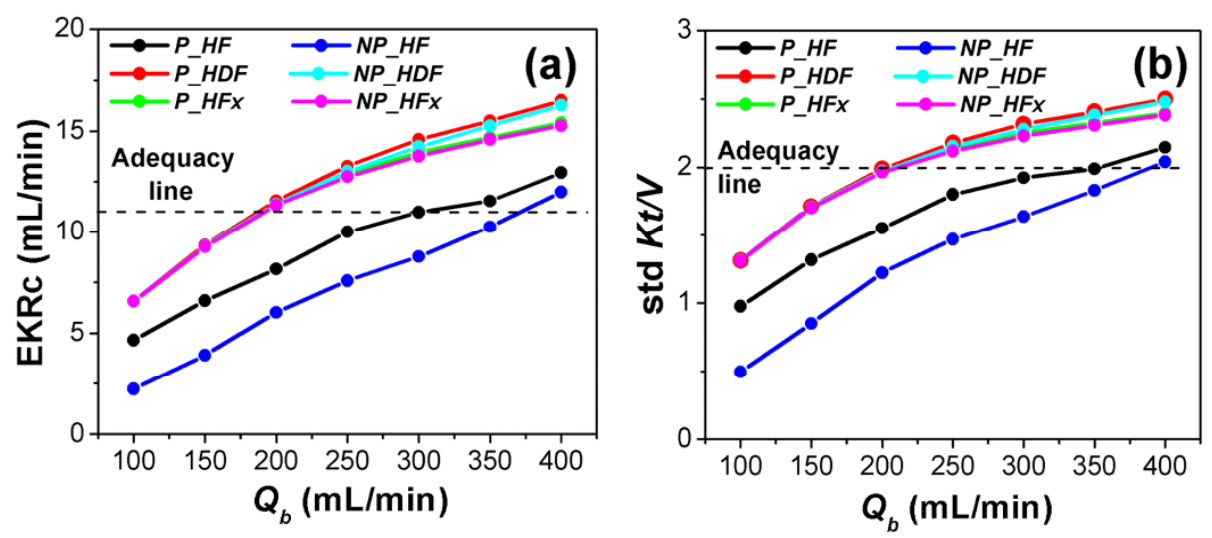

Figure 5 Comparison of treatment doses; EKRc and std $\boldsymbol{K} \boldsymbol{t} / \boldsymbol{V}$. This shows the variations of (a) EKRc and (b) std $K t / N$ according to the mean blood flow rate under various treatment conditions and with various pump types. $Q_{b}$ indicates the mean blood flow rate, $P_{-} H F, P_{-} H D F$ and $P_{-} H F x$ are pulsatile HF, HDF and HFx, respectively, and NP_HF, NP_HDF and NP_HFx are non-pulsatile HF, HDF and HFx, respectively. 
and delivered treatment dose to patients with CRF (Figure 5) for various blood purification therapies, i.e., HF, HDF and HFx.

HF treatment provides only convective toxin removal by UF and injection of replacement fluid, whilst the HDF and HFx treatments provide diffusive toxin removal driven by concentration differences between the blood and dialysate as well as convective toxin removal. The convective toxin removal is dependent on the UF rate and sieving coefficient. The in vitro experiment demonstrated that pulsatile pumping improved the UF rate by increasing the UF coefficient and the TMP (Figure 2). The improved UF rate increased the convective toxin removal. Due to the effect of diffusive clearance, the contributions of convective toxin removal to the total clearance in the HDF and HFx treatments were lower than that in the HF treatment. This explains why the increased clearance by pulsatile pumping showed less benefit in the HDF and HFx treatments than in the HF treatment as shown in Figure 4.

The sieving coefficient of the dialyser for B2M was 0.8 , whereas the value for urea was 1. These observations indicated that in the HF treatment, B2M removal was $20 \%$ less than that of urea under the same UF rate conditions. Thus, the convective clearance for B2M was $20 \%$ less than that for urea in HF during which convective toxin clearance occurred. However, HDF and HFx use the mechanisms of diffusive and convective toxin removal simultaneously. In these cases, B2M removal was $20 \%$ less than that of urea by the convective effect under the same UF rate conditions. In contrast, the diffusive mass transfer coefficient of the dialyser for B2M [KoA ${ }_{B 2 M}$ in Eq. (A8) in the Appendix] was $70 \%$ less than that for urea, which exceeded the $20 \%$ decrease compared to urea by the convective effect. Therefore, in the case of HDF and HFx, the relative portion of convective toxin removal to diffusive toxin removal for B2M was more remarkable than that for urea. These observations explain why the percentage increase in total clearance by pulsatile pumping for B2M was more remarkable than that for urea, as shown in Figure 4.

To test the long-term performance of the three blood purification therapies and predict the effects of pump type on their performance, we simulated the therapies for 5 weeks and obtained the EKRc and std $K t / V$ indices for each case (Figure 5). In terms of the EKRc and std $K t / V$ indices, HDF therapy showed the best performance, followed by HFx therapy. HF therapy showed the poorest performance because it provided only convective toxin removal, whilst the others provided convective and diffusive toxin removals using the dialysis solution. However, the pulsatile effect on the delivered dose was best in HF therapy, with neither HDF nor HFx showing a remarkable effect. As shown in Figure 5 , the increases in delivered treatment doses of about $25 \%$ for both EKRc and std $K t / V$ in HF therapy suggested that use of a pulsatile pump may become a practical alternative to the conventional method with a non-pulsatile pump for HF treatment.

Although we developed a mathematical model of a CRF patient to assess several blood purification methods, this model is not limited to chronic conditions. It can be used to model an acute renal failure patient by modifying the mass transfer coefficients and considering residual renal function.

Several animal studies have shown that a blood purification system using a pulsatile blood pump can effectively maintain a physiologically stable hemodynamic state, i.e., the heart rate, arterial blood pressure, and hematocrit, and is a plausible alternative to systems using a conventional roller pump [10-12]. Furthermore, our in vitro experiment showed that the hematocrit was maintained within the normal range during pulsatile 
pumping. This means that no blood damage occurred due to the pulse peak pressure during pulsatile blood purification therapy.

The use of a pulsatile blood pump in a blood purification system with a semipermeable membrane was suggested to increase convective toxin removal, which could reduce both treatment time and associated costs.

Although we provided simulated results for the test of the effects of pulsatile pump on toxin removal, there are some limitations to our study. First, we have validated our numerical patient model by comparing the simulated results with that the clinical test only using a conventional non-pulsatile system. However, a comparison with the experiment using pulsatile pump was not attempted because of no available pulsatile pump that can be applied to patients among commercialized blood pumps. In addition, the input parameters of the model, such as the pressures at the dialyser inlet and outlet and UF coefficients, were estimated from in vitro rather than in vivo experiment.

\section{Conclusions}

We proposed a new mathematical model to assess the toxin removal efficiency of blood purification systems in patients, integrating the mass transfer model for a human body with a dialyser. In vivo experiments were also performed to verify clinically the model results, respectively. Using the verified model, we simulated the effect of pulsatile pumping on convective toxin clearance and delivered treatment dose to patients with CRF for various blood purification therapies. Compared with non-pusatile pumping method, the increases of urea and B2M clearances by pulsatile pump were highest in HF treatment (122.7\% and $122.7 \%$, respectively), followed by HDF (3.6\% and $8.3 \%$, respectively) and HFx (1.9\% and 4.7\%, respectively). EKRc and std $K t / V$ were on average $28 \%$ and $23 \%$ higher, respectively, in the pulsatile group than in the non-pulsatile group in HF treatment.

The superiority of pulsatile pumping is significant in HF treatment, which relies only on convection for toxin removal. In both HDF and HFx treatments, the effectiveness of pulsatile pumping on B2M removal, which depends more on convection compared to urea removal, was more remarkable than that on urea removal. Finally, pulsatile system will help any blood purification treatments, whose mechanism of toxin removal is mainly convection, get better performance than conventional non-pulsatile system.

\section{Appendix}

\section{A1. In vitro experiment for identification of model parameters}

A schematic of the experiment is shown in Figure 6. The experiments were performed for a pulsatile pump (T-PLS; BHK Inc., Seoul, Republic of Korea) and a non-pulsatile pump (AK95 roller pump; Gambro Inc., Hechingen, Germany) under various flow rate conditions from 100 to $400 \mathrm{~mL} / \mathrm{min}$ in increments of $50 \mathrm{~mL} / \mathrm{min}$. Figure 7 shows the pressure waveforms at the dialyser inlet generated by the two pumps. The AK95 pump generated a very weak pulsatile flow that can be considered as almost non-pulsatile flow. Each test was performed using bovine whole blood (heparin of $16000 \mathrm{IU} / \mathrm{L}, \mathrm{Hct}=31 \%$, $\mathrm{T}=37^{\circ} \mathrm{C}$ ). In the dialysate circuit, we used the AK95 roller pump at a constant flow rate of $300 \mathrm{~mL} / \mathrm{min}$ for all test cases. A polysulphone dialyser (FX60; FMC Inc., Frankfurt, Germany) was used with normal saline as the dialysis solution. An ultrasonic flow sensor (T109; Transonic Systems Inc., Ithaca, NY) was placed in the blood circuit before the dialyser to measure the flow rate. Four pressure sensors (Pressure transducer; Sensys 
Inc., Seoul, Republic of Korea) were inserted before and after the dialyser in the blood and dialysate circuits. In the experiment, according to the pump type and flow rate, we measured the four pressures (at the dialyser inlet and outlet of the blood circuit, and at the dialyser inlet and outlet of the dialysate circuit) and UF coefficient of the dialyser. Before starting the test, we recirculated bovine blood through the dialyser at $200 \mathrm{~mL} /$ $\min$ for $2 \mathrm{~h}$ to develop a sufficient protein layer as suggested in a previous study [13].

The goal of the in vitro experiment was to establish reference data for the variation in TMPm and UF coefficient according to pump type and flow rate. Thus, the parameters such as TMPm and the UF coefficient for a specific condition of flow rate and pump type can be obtained by interpolating the reference data.

\section{A2. Governing equations of the mathematical model}

The time derivatives of the intracellular fluid $\left(V_{i c}\right)$, interstitial fluid $\left(V_{i s}\right)$ and plasma $\left(V_{p l}\right)$ can be expressed as

$$
\begin{aligned}
& \frac{d V_{i c}}{d t}-k_{f}\left[O_{i c}(t)-O_{i s}(t)\right] \\
& \begin{aligned}
\frac{d V_{i s}}{d t}= & -k_{f}\left(O_{i c}(t)-O_{i s}(t)\right) \\
& +F_{a}(t)-R_{v}(t)
\end{aligned} \\
& \frac{d V_{p l}}{d t}=0-F_{a}(t)+R_{v}(t)-Q_{f}
\end{aligned}
$$

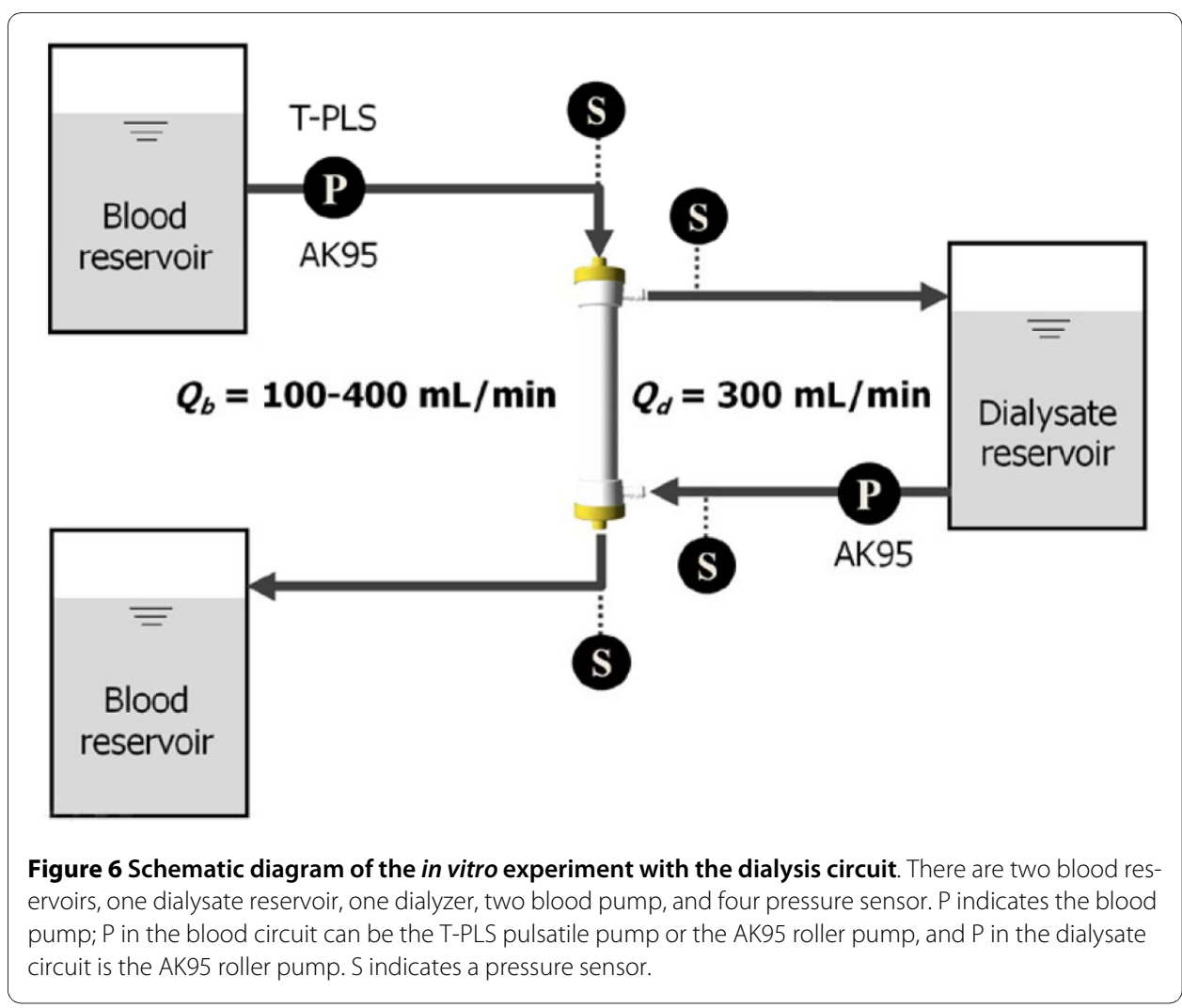




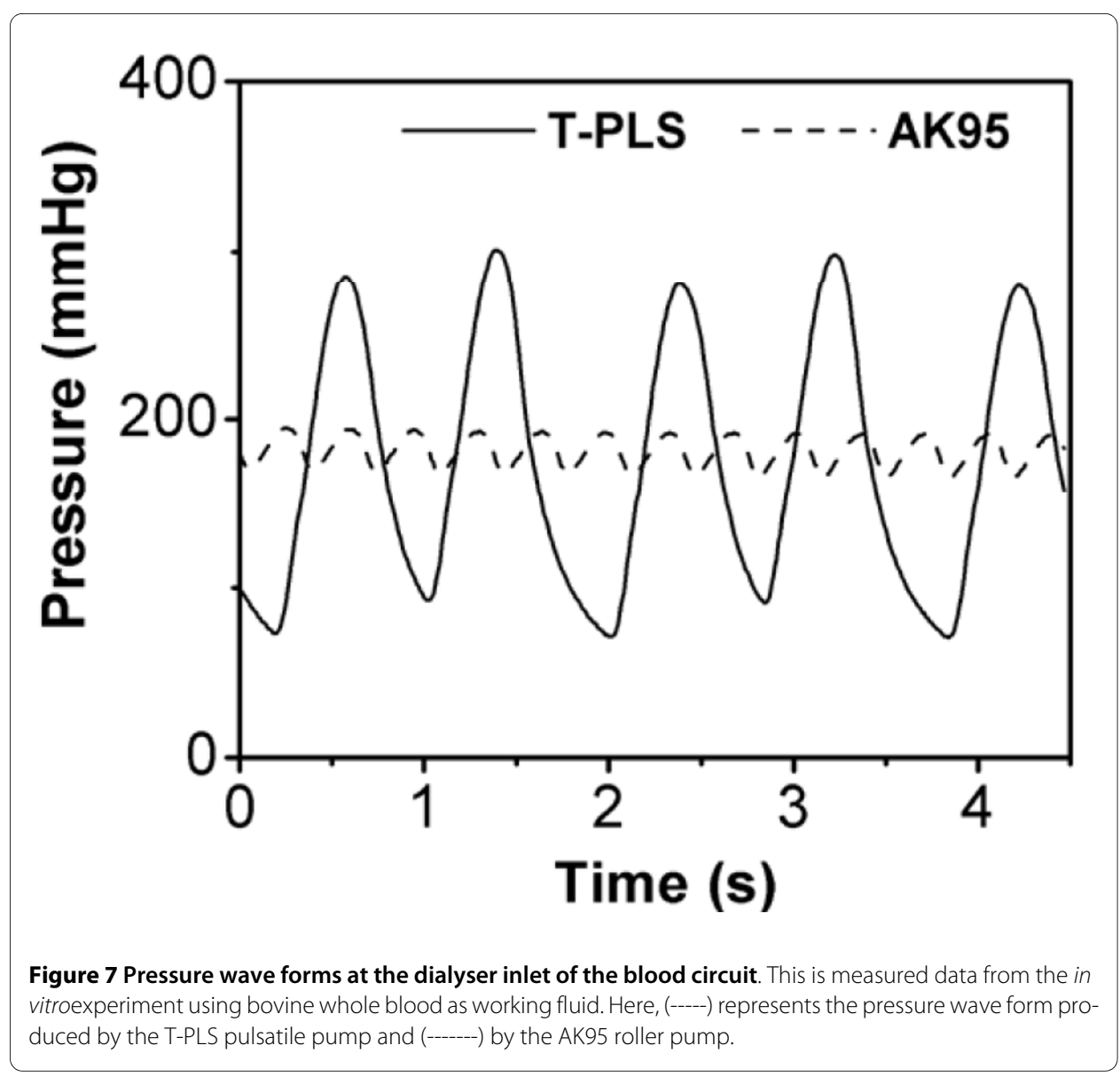

where $k_{f}$ is the osmotic filtration coefficient at the cellular membrane, $O_{i c}$ and $O_{i s}$ are the osmotic concentrations of the intracellular and interstitial compartments, respectively, $F_{a}$ is fluid filtration rate at the arterial capillaries, $R_{v}$ is the fluid reabsorption rate at the venous capillaries and $Q_{f}$ is the UF rate.

The time derivatives of the solute concentrations in the intracellular $\left(M_{s, i c}\right)$ and extracellular $\left(M_{s, e x}\right)$ compartments are expressed as follows:

$$
\begin{aligned}
\frac{d M_{s, i c}}{d t}= & -\eta_{s}\left(C_{s, i c}(t)-\beta_{s} C_{s, e x}(t)\right) \\
\frac{d M_{s, e x}}{d t} & =\eta_{s}\left(C_{s, i c}(t)-\beta_{s} C_{s, e x}(t)\right) \\
& +G_{s}-J_{s}(t)
\end{aligned}
$$

where $\eta_{s}$ is the mass transfer coefficient of solute $s$ at the cellular membrane, $\beta_{s}$ is the equilibrium ratio of solute $s, C_{s, i c}$ and $C_{s, e x}$ are the intracellular and extracellular concentrations of solute $s$, respectively, $G_{s}$ is the generation rate of solute $s$ and $J_{s}$ is the solute transfer rate through the dialyser membrane. We assumed $G_{\text {urea }}$ to be $6.24 \mathrm{mg} / \mathrm{min}$ [2], and $J_{s}$ is derived below: 


$$
\begin{aligned}
J_{s}(t)= & Q_{f}(t) \frac{1}{r} C_{s, p l}(t)-K_{s}\left(1-\frac{Q_{f}(t)}{Q_{b}}\right) \\
& \left(\frac{\alpha_{s}}{r} C_{s, p l}(t)-C_{s, d}(t)\right)
\end{aligned}
$$

where $\alpha_{s}$ is the Gibbs-Donnan equilibrium ratio of solute $s, r$ is the plasma water fraction and $K_{s}$ is the clearance of solute $s$. HDF or HFx treatments use simultaneous diffusion and convection for toxin removal. Thus, toxin clearance is calculated as the sum of diffusive clearance $\left(K d_{s}\right)$ and convective clearance $\left(K c_{s}\right)$ as follows:

$$
K_{s}=K d_{s}+K c_{s}
$$

The diffusive clearance is expressed as

$$
K d_{s}=Q_{b}\left[\frac{e^{K o A_{s}\left(\frac{Q_{d}-Q_{b}}{Q_{d} Q_{b}}\right)}-1}{e^{K o A_{s}\left(\frac{Q_{d}-Q_{b}}{Q_{d} Q_{b}}\right)}-\frac{Q_{b}}{Q_{d}}}\right]
$$

where $K o A_{s}$ is the diffusive mass transfer coefficient of the dialyser for solute $s$ and $Q_{b}$ and $Q_{d}$ are the blood and dialysate flow rates through the dialyser, respectively. The convective clearance is expressed as

$$
K c_{s}=Q_{f} S_{i} T
$$

where $S i_{s}$ is the membrane sieving coefficient of solute $s . T$, termed the transmittance, represents the $\mathrm{mL} / \mathrm{min}$ increase in clearance for each $\mathrm{mL} / \mathrm{min}$ of filtration and can be calculated as follows:

$$
T=1-\frac{K d_{s}}{Q b}
$$

The HF treatment uses only convection for toxin removal, and thus the convective clearance is simply calculated as follows:

$$
K c_{s}=Q_{f} S i_{S}
$$

More detailed equations were described previously by Ursino et al. [6] and Depner and Garred [2].

\section{A3. Derivation of the internal UF rate for the HFx treatment}

To calculate the internal UF rate in the HFx treatment model, we introduce the UF coefficient per unit length, which is determined by dividing the UF coefficient by the dialyser length as follows: 


$$
K_{u f l}=K_{u f} / L
$$

where $K_{u f l}$ is the UF coefficient per unit length and $L$ is the dialyser length. As a simplified approximation, the local pressure distributions along the dialyser fibres in the blood and dialysate circuits are described as follows:

$$
\begin{aligned}
& P_{b}(x)=\frac{\left[P_{b, \text { in }}-P_{b, \text { out }}\right]}{L} x+P_{b, \text { in }} \\
& P_{d}(x)=\frac{\left[P_{d, \text { in }}-P_{d, \text { out }}\right]}{L} x+P_{d, \text { in }}
\end{aligned}
$$

where $x$ indicates the local position along the dialyser, $P_{b}(x)$ and $P_{d}(x)$ are the mean pressures at local position $x$ in the blood circuit and dialysate circuit, respectively. The UF rate, which is the primary determinant of the convective clearance of the toxin, is calculated as follows:

$$
Q_{f}=\int_{0}^{M} K_{u f l} \times\left[P_{b}(x)-P_{d}(x)\right] d x
$$

where $M$ is a local point where neither UF nor backfiltration is generated. The internal UF rate is directly related to the convective toxin clearance in the HFx treatment, and therefore, the convective clearance for the HFx treatment is calculated using Eq. (A9) in the Appendix.

\section{A4. Definition of criterion indices}

The time-averaged concentration of solute $s\left(T A C_{s}\right)$ is calculated by integrating the concentration profile of the solute in the plasma compartments with respect to time as follows:

$$
T A C_{s}=\frac{1}{t} \int_{0}^{t} C_{s, p l}(t) d t
$$

Equivalent renal clearance $\left(E K R_{s}\right)$ is calculated as follows:

$$
E K R_{s}=\frac{G_{S}}{T A C_{S}}
$$

$E K R c_{s}$ is the corrected value of $E K R_{s}$ for the normalised water volume, $40 \mathrm{~L}$, as follows:

$$
E K R c_{s}=\frac{E K R_{s}}{V} \times 40
$$

where $V$ is the total body fluid.

The mean pre-treatment concentration of solute $s\left(M P C_{s}\right)$ is acquired by averaging the pre-treatment concentrations in the plasma compartment at steady state, and the weekly std $K t / V$ is then calculated as follows: 


$$
\text { std } K t / V=\frac{G_{\text {urea } \times t}}{M_{\text {Prea }} \times V}
$$

where $t$ is the total time. All equations are adapted from Ursino et al. [6] and Depner and Garred [2].

\section{Competing interests}

The authors declare that they have no competing interests.

\section{Authors' contributions}

KML carried out all the computations and analyzed the computed data. EBS designed the computations, analyzed and interpreted the computed data. All authors were actively involved in the writing of the manuscript, read it and approved the final manuscript.

\section{Authors' information}

KML received Ph.D. from Dept of Biomedical Engineering of Seoul National University, South Korea at 2008 and thereafter worked for the Biosystems Engineering Lab of Kangwon National University, South Korea, as a post doc. And now he is working for the department of Biomedical Engineering of Johns Hopkins Univ. as post doc.

EBS finished undergraduate course at Seoul National University and received Ph.D. from the Mechanical Engineering Dept. of KAIST (Korea Advanced Institute of Science and Technology) at 1994. He received another Ph.D. at the Dept. of Physiology (Medical School) of Kyoto University, Japan, at 2008. He became a full professor of Kangwon National University from 2004.

\section{Acknowledgements}

This work was supported by the NRL (National Research Lab) program of National Research Foundation of Korea (No. ROA-2008-000-20127-0), by the Korea Research Foundation Grant funded by the Korean Government (MEST) (The Regional Research Universities Program/Medical \& Bio-Materials Research Center), and by the National Research Foundation of Korea (No. 313-2007-2-D00968).

\section{Author Details}

Department of Mechanical \& Biomedical Engineering, Kangwon National University, Chuncheon, Kangwon-do 200-701, Republic of Korea

Received: 31 January 2010 Accepted: 22 June 2010

Published: 22 June 2010

\section{References}

1. Ronco C: Hemofiltration and hemodiafiltration. In Hemodialysis High-Efficiency Treatments Edited by: Bosch P. San Antonio, Churchill Livingstone; 1993:119-133.

2. Depner T, Garred L: Solute transport mechanisms in dialysis. In Replacement of Renal Function by Dialysis Edited by: W Horl W, Norwell. Kluwer Academic Publishers; 2004:73-93.

3. Lim KM, Park JY, Lee JC, Kim JC, Min BG, Kang ET, Shim EB: Quantitative analysis of pulsatile flow contribution to ultrafiltration. Artif Organs 2009, 33(1):69-73.

4. Runge TM, Briceno JC, Sheller ME, Moritz CE, Sloan L, Bohls FO, Ottmers SE: Hemodialysis: evidence of enhanced molecular clearance and ultrafiltration volume by using pulsatile flow. Int J Artif Organs 1993, 16(9):645-652.

5. Cameron JS: Dialysis arthropathy, amyloidosis and beta-2 microglobulin. Pediatr Nephrol 1987, 1(2):224-229.

6. Ursino M, Coli L, Brighenti C, Chiari L, de Pascalis A, Avanzolini G: Prediction of solute kinetics, acid-base status, and blood volume changes during profiled hemodialysis. Ann Biomed Eng 2000, 28(2):204-216.

7. Jaber BL, Zimmerman DL, Teehan GS, Swedko P, Burns K, Meyer KB, Leypoldt JK: Daily hemofiltration for end-stage renal disease: a feasibility and efficacy trial. Blood Purif 2004, 22(6):481-489.

8. Yamashita AC, Kawanishi H: Kinetics and dose of daily hemofiltration. Blood Purif 2004, 22:14-19.

9. Lee JC, Park CY, Choi SW, Park JY, Kim JC, Yun YM, Lee KK, Sun K, Min BG: Computational dose predictions for combined treatment of hemofiltration with weekly hemodialysis. Comput Methods Programs Biomed 2008, 89(3):275-281

10. Lopez-Herce J, Ruperez M, Sanchez C, Garcia C, Garcia E, Rodriguez D, Del Canizo JF: Continuous venovenous renal replacement therapy with a pulsatile tubular blood pump: analysis of efficacy parameters. Artif Organs 2006 30(1):64-69.

11. Ruperez M, Lopez-Herce J, Sanchez C, Garcia C, Garcia E, Del Francisco CJ: Comparison of a tubular pulsatile pump and a volumetric pump for continuous venovenous renal replacement therapy in a pediatric animal model. ASIO J 2005, 51(4):372-375.

12. Ruperez M, Sanchez C, Garcia C, Garcia E, Lopez-Herce J, Del Canizo FJ, Vigil D: Continuous venovenous renal replacement therapy using a pulsatile blood pump. Pediatr Nephrol 2003, 18(1):29-32.

13. Morti SM, Zydney AL: Protein-membrane interactions during hemodialysis: effects on solute transport. ASAIO J $1998,44: 319-326$.

doi: 10.1186/1475-925X-9-31

Cite this article as: Lim and Shim, Computational assessment of the effects of a pulsatile pump on toxin removal in blood purification BioMedical Engineering OnLine 2010, 9:31 